Mark Wilberforce, Jane Hughes, Paul Clarkson, David Whyte, Helen Chester, Sue Davies, David Challis

An electronic referral system supporting integrated hospital discharge.

Journal of Integrated Care Vol. 25 No. 2, pp. 99-109.

https://doi.org/10.1108/JICA-09-2016-0034 


\section{An electronic referral system supporting integrated hospital discharge.}

\section{Introduction}

A long-standing concern both in the UK and internationally is that multiple health and social care professionals may undertake assessments for older people needing community support for complex needs, but that this information is not shared (Challis et al., 2010). The consequences include economic waste where collected information is duplicated, and a missed opportunity to share data for coordinated care planning that may facilitate swifter hospital discharge and reduced use of residential care (Abendstern et al., 2008; Byles, 2000; Clarkson et al., 2009; DH, 2013; Scott and Hawkins, 2008). Patients may experience a conveyor-belt care system where they move between siloed services, causing frustration and anxiety.

Government attempts to address these shortcomings include the Single Assessment Process (SAP) in the UK from 2004, which sought to deliver an architecture for both 'core' and 'specialist' assessments to be undertaken using a common structure, with the aim of increasing their portability across professionals and agencies (DH, 2001). More recent administrations have continued the theme, with the Better Care Fund seeking to extend pooled budget arrangements between health and social care authorities; and the provision of care coordination and care plans with a named individual to oversee care for people with long-term complex health and social care needs (DH, 2013, DH and DCLG, 2014). Attempts to integrate assessments have also aimed to build on new technologies, aligned with the government's ambition for a new NHS IT infrastructure so that information recorded at first contact with a professional can be shared to improve care and service arrangement $(\mathrm{DH}, 2012)$.

The Common Assessment Framework (CAF) for adults was introduced as a broad policy intending to "facilitate more efficient, timely and secure exchange of information around assessments, support and care planning" (DH, 2009; p8). Drawing on similar principles used in the CAF for children and young people, the objectives included an explicit intent to introduce a secure and effective means of sharing electronic records across health and social care organisations. In 2009 the Department of Health awarded 11 local authority-led partnerships (termed 'demonstration sites') central 
government funding as part of the CAF policy, with each encouraged to test different initiatives in different settings, but all oriented towards meeting the policy's broad aims (Chester et al., 2015).

The genesis of the CAF can be linked to a much older debate around the role of assessment in care coordination, and two inter-linked processes: case finding and screening (Abell et al., 2010; Raiff and Shore, 1993). Case finding is intended to ensure that a high proportion of those for whom a service is appropriate have the opportunity to receive it. Conversely the case screening process is designed to ensure that only those service users whose needs and circumstances meet the service's eligibility criteria receive a more comprehensive assessment (Challis and Davies, 1986). However, there is less agreement about how information should be collected, stored, and transmitted, and the extent to which care coordinators or hospital discharge planners are involved (Scott and Hawkins, 2008). This paper reports evaluation findings relating to one CAF demonstration site which tested a new electronic hospital discharge system to support the subsequent community care of older adults with complex needs.

\section{The pilot}

The pilot was a joint enterprise between an acute hospital NHS Trust in the north-west of England and the corresponding local authority. The original pilot design centred on the on-site Integrated Discharge Team (IDT). The IDT comprised discharge coordinators (including nurses, social workers and social care assessors) who undertook needs assessment and coordinated primary health care and local authority services to enable prompt and safe discharge. The IDT supported the discharge of only a minority of patients, whose particularly complex needs required careful coordination. The original pilot design comprised a significant change to a largely paper-based process in the way the IDT both received and made referrals, and how associated patient information was shared.

With respect to receiving referrals, the prevailing system required hospital ward staff to hand-complete paper proformas, which were physically collected from each ward on a daily basis by IDT staff. The pilot aimed to test a new electronic referral system which had the potential to improve the quality and adequacy of information needed for 
the IDT to make its decisions, and reduce the time taken. As part of the pilot, a ward nurse making a referral to the IDT would first open 'ShareCare'; a local authority electronic records system accessible from hospital computers. The pilot introduced a new module within ShareCare that enabled direct referrals to the IDT. This module was pre-populated with a core information set already recorded in ShareCare (drawn, in turn, from the national NHS 'spine' system) intended to save time and improve accuracy. Ward staff would complete other elements of the module, providing details of the admission and likely care needs, and requesting IDT involvement in discharge planning. By saving and submitting the form, the ShareCare system automatically alerted the IDT to the new referral which would then be discussed (as with regular paper-based referrals) at daily meetings. Assessments and necessary planning would then be initiated.

In terms of onward referrals, the pilot initially intended for the ShareCare module to enable IDT staff to share its assessment information with a wider number of services, and make referrals to community services identified in its discharge plans. By including primary care as a project partner, referrals and information sharing could be extended to community nursing, intermediate care and other services for people with long-term conditions. Similar arrangements were also planned for palliative care services. This would reduce duplication of information in making referrals to these services using separate proformas and enable multiple referrals to be made simultaneously.

The research aimed to explore the implementation and potential value of the new system. Specifically, it addressed four research questions:

1. What was the practitioner experience of using the pilot system, and perceptions of strengths and limitations?

2. Did the pilot reduce the time taken to make referrals?

3. What were the characteristics of those referred through the new system?

4. Did the pilot improve the quality of information on referrals?

\section{Method}

Data collection 
A mixed methods approach was adopted comprising two sources of data collection. First, semi-structured interviews were undertaken with 7 members of staff involved in the pilot. Interviews were conducted with two nurses acting as Discharge Coordinators in the IDT; two nurses from the participating wards; one social care assessor; one local authority social worker; and the Discharge Service Manager. The research team developed a topic guide using themes identified in a prior review of the determinants of success in clinical information systems (Van der Meijden et al., 2003). Interviews thus spanned system quality (e.g. ease of access and security); information quality (e.g. accuracy and completeness); usage (e.g. whether process took less time to complete); staff satisfaction (e.g. how well new system fit with existing work patterns) and impact (e.g. how well system worked alongside other technology). Interviews lasted up to 2 hours, and were undertaken during May and June 2012 (shortly after the pilot ended).

To support the qualitative data in relation to Research Questions 3 and 4, a second data collection exercise in Autumn 2012 extracted administrative records for referrals made from participating hospital wards and the IDT during the pilot. A pragmatic natural experiment was undertaken. Selected staff were trained in the use of the new referral system and used it during the pilot period to refer patients to the IDT. Other ward members continued to use paper-based forms. Information was collected on all referrals from the participating wards to the IDT during the pilot. The data included patient socio-demographic characteristics, physical functioning (activities of daily living); whether the patient had impaired cognition; whether they had any prior admissions; details of the current admission (time, date, specialty of ward, moves between specialities, and whether referred to psychiatrist); and discharge information (date of discharge and post-discharge care arrangements).

\section{Data analysis}

A theoretical, or deductive, thematic analysis of audio-recordings from the interviews was conducted by repeatedly playing the recordings which were then summarised under the under the thematic headings used in the topic guide. This was then supplemented with documentary evidence where factual information (especially in 
relation to technical features of the technology) could be ascertained from such material.

The quality of referral information was assessed by examining the number of items that were missing data relevant to the discharge. Due to circumstances beyond the researchers' control (described within the Findings) the final sample size was modest. Simple descriptive analyses in STATA were then used to compare sample characteristics and data quality between those referred by paper and electronic processes. The research received ethical approval from the NHS National Research Ethics Committee (South West, Bristol) on 23rd November 2010 (reference: 10/H0107/60).

\section{Results}

The results begin with an overview of implementation, which establishes the context in which the other findings should be viewed. The research questions are then addressed in order.

\section{An overview of implementation}

Prior to the start of the pilot's operational phase, two decisions were taken that considerably altered the original plans. First, one of the NHS partners formally withdrew from the CAF partnership. In terms of outgoing referrals, plans to allow referrals to a broad range of community-orientated services were therefore shelved and practice continued as before. Second, the local authority decided to discontinue its IT supplier, and instead switch to an alternative provider meaning that the ShareCare module would operate for a severely restricted length of time. Consequently, the number of participating hospital wards was reduced to just two (Orthopaedic and Care of the Elderly), and training restricted to core staff on each ward who would use the ShareCare IDT module during the pilot. The ShareCare module ceased operating after just five weeks, and was only used to refer 22 patients during the pilot from hospital wards to the IDT (whilst 51 were referred during the same period from the same wards using conventional paper proformas).

\section{Practitioner and manager experience}


The interviews initially explored the local context and perceptions of existing systems. They identified that, before the pilot, the use of IT in making referrals across hospital wards to other hospital-based services, and to allied community services, was extremely limited. Although some wards were reported to make referrals to Intermediate Care using the ShareCare system, this was considered an exception rather than a rule. Overwhelmingly, referrals were through proformas transmitted by post, by fax or hand-delivered by staff. Several concerns were noted, the most obvious being the administrative and time costs of having to duplicate hand-written information, and especially where more than one referral may be necessary to different services. Accuracy and security of data were also highlighted. Less obvious was a frustration that referring practitioners could not monitor the subsequent progress of their referrals and whether actions had been taken, which was perceived as largely 'hidden'. Ward staff reported having to continually telephone colleagues to ascertain progress with regard to post-discharge arrangements for patient care.

\section{Impact on referral time}

The ShareCare module was viewed as requiring more time to submit a referral than paper proformas, since the latter were reported to be relatively well designed. The Discharge Coordinators estimated that they could complete a paper form, on average, in less than five minutes per case. However, loading the ShareCare system was reported to take, on average, 15 minutes per case and required the inputter to move backwards and forwards within the system, remembering to "refresh" and "save" it at each stage or else the data would be lost. The ShareCare module was described as unwieldy in terms of speed. Recalling the paper proformas, one respondent said:

"It was a few pages...[There's] actually a sticker with the patient's details on, address, date of birth, that sort of thing, and then reason for admission, doctor that they're under, date they came in. And then ... you just literally circle options, so it's much quicker... Anyone can fill in the form and then hand it over to IDT, whereas on the [ShareCare module] you've got to have access...it's computers that are the problem". 
It was suggested that it would have been impractical to expect a busy nurse, for example, to use the system because they would not have the time required in one block. Yet when a user logged out of the system to complete the task at a later time, they would be returned to the start of the system and have to scroll through again to the point at which they had been working. IDT members on occasions had anxiety about whether their data had actually been saved.

Practitioners in the IDT felt that the idea of this system was good but that the implementation was problematic and "cumbersome". Although the ShareCare module eradicated the need for a paper proforma, it was described as mis-fitting and like a "square peg in a round hole". The "clumsiness" led to people having to duplicate what the system did in other ways. For example, one respondent said:

"The only way you can describe it is...it was supposed to be if you imagine your computer system as a filing cabinet, you were supposed to have one piece of paper in that system where everybody could, sort of, go in do what they wanted and bob back out, but actually what you got was a filing cabinet with a lot of separate things in it...it wasn't opening the filing cabinet and seeing the full picture, it was opening the filing cabinet and starting again."

IDT members also perceived that the ShareCare module did not readily identify their referrals. They had to work through four on-screen pages to reach this and given the system was considered slow, this took time. Practitioners were also asked whether the introduction of the ShareCare module brought about any additional requirements particular to the system. Respondents noted that it required an additional 'hospital' login since it sat on the local intranet. This meant that, potentially, any NHS staff with requisite credentials could use the system, but not local authority staff requiring access.

Paradoxically, the system was also considered to have generated more paper than before since meetings still required information to be printed out so information could be discussed collectively. It was considered impractical to undertake these meetings around computer screens. However, IDT members did express a preference for the 
electronic system in terms of data security, perceiving it could avoid some of the hazards of handwritten notes.

\section{Patient characteristics}

Table 1 provides an overview of patient characteristics referred to the IDT during the pilot period from the two participating wards. The data revealed a high degree of dependency relative to other studies of hospital discharge (for example see Challis et al., 2012), and reflects the intention of this initiative in targeting complex cases. The data also revealed a wide range of health problems as identified by primary diagnostic codes (ICD10). Diseases of the circulatory system were the most common diagnosis $(n=16)$, followed by unclassified symptoms ( $n=14$, with syncope and senility being the most prevalent); a range of injuries, including fractures most likely associated with falls $(n=9)$; and illness of the gastro-urinary system $(n=7)$. Those referred through the ShareCare module and paper proformas appeared relatively balanced with respect to age, gender, receipt of informal care and features of their admission. However, those referred through the ShareCare module were significantly more likely to have been in receipt of home care on admission, and were less likely to have been moved between two or more specialties during their hospital episode. Additionally, there was no indication that the method of referral to IDT was linked to either length of stay, domicile on discharge or composition of care plan arrangements.

\section{[Table 1 about here]}

\section{Impact on information quality}

Information quality was assessed in two ways. First, for the 72 referrals to the IDT included in this study, the quality of information could be assessed according to the completeness of the available data. A simple 'count' of missing data items was calculated for 37 fields of data items (thus 2664 data items possible for 72 patients) returned from the Trust. In total 16 percent of all requested data items were missing, and this rate was greater for those patients referred through the ShareCare system than through paper proformas ( 21 vs 13 percent respectively, $p=0.039$ ). Table 2 presents missing data by broad category. The largest source of missing data related to dependency characteristics, with 44 percent missing data amongst those referred using the new technology. 
[Table 2 about here]

Second, findings from the interviews revealed that the aspiration to pre-populate the ShareCare module from the NHS "Spine" was often not achieved. At times, patients were not on the NHS Spine and so the Discharge Coordinators would be required to populate all aspects, and in other examples many items were missing. On occasion, the pre-populated information was incorrect (e.g. out of date) but the system allowed the Discharge Coordinators to amend it. It was further reported that there was no space available for other relevant information - such as number of carers and details of existing care package. The technology was not an exact replica of the paper form that was used previously and staff could not add many notes or any other information.

There was some suggestion that the ShareCare module could become more accurate than paper-based systems because of the capacity to update information over time. A paper proforma was only as recent as the date it was written, but the ShareCare module could be amended and saved as required. As such, it was anticipated that the benefits of the pilot in terms of information quality could be accrued over time:

"It's the buy-in of the first person having to put so much information in the first time. But then for subsequent users, it speeds it all up because you're getting information out while you're still doing your assessment and putting it on ...in theory it's fantastic".

Respondents further perceived that the ShareCare module permitted a clear and continuous record, which could form an audit trail, and allow anyone to track progress with the referral.

Some respondents additionally reflected on what was necessarily lost in conveying information electronically, rather than in person. Previously, respondents reported that they often discussed their referrals with a member of the IDT at the point that they picked them up (commonly on the wards themselves), enabling them to discuss a patient's circumstances and emphasise any point of importance. However the IT 
transmission of information meant the opportunity to amplify the information on the referral was lost.

\section{Discussion}

The UK health and social care systems have long-struggled with developing means for standardising approaches to information collection and data sharing, especially for the purpose of targeting services appropriately and screening for needs amongst frail older populations. The value of shared assessment information can be traced at least to the emergence of care management as a means for improving community support (Abell et al., 2010; Taylor 2012), but a concerted government effort to make further strides is evident from 2000 through the SAP and many other reforms that sought to improve integrated working. Whilst these efforts continue in England, clear parallels can also be drawn from devolved administrations, such as the drive to improve coordination and person-centredness in dementia assessment in Scotland (Scottish Government, 2013). Furthermore, services in Northern Ireland have played an important role in testing new shared assessment protocols (Taylor, 2012). Yet progress remains slow, and the possibility for new IT technology to enhance information recording and transmission has been pursued across UK health systems at much expense. Thus, whilst the individual CAF initiative reported here may be relatively small, it can be seen as part of a much wider context of reform.

The new technology provided some evidence of 'proof-of-concept': that is, outside of the particular implementation challenges, practitioners could nevertheless see indications of the benefits of electronic referral and information sharing. Further work would, however, be required if the system was re-instated to ensure that it reflected the requirements of the Care Act 2014 (TLAP, 2014). Other research has commented on the importance of shared assessment systems for the design of successful postdischarge care plans for older people (Taylor, 2012) and their value in countering the silo-culture common to many hospital departments (Scott and Hawkins, 2008). The IDT system was seen as having the potential to eliminate the duplication of written information already held electronically, and would be more secure and confidential than paper-recorded information - findings that echo concerns with paper-based information sharing used in some single assessment processes (Dickinson, 2006). A further efficiency was to eliminate the need to 'chase' progress with a referral, since 
actions could be viewed on screen. These positive reports echo Waterson's (2014) case study that the introduction of technological support to early discharge planning for hospitalised stroke patients was routinely welcomed by practitioners, despite implementation challenges.

However, the initiative was largely unsuccessful when judged against its broader objectives. There were technology-design issues that contributed to a 'clunky' interface and lack of usability, preventing ward practitioners from easily completing the proformas, and IDT members from identifying their referrals. Furthermore, even accounting for the limited time to become familiar with the new technology, the ten minute differential between the reported time to complete the electronic (vs paper) referral appears to represent an inefficient process. There was also some evidence that electronic referrals were associated with reduced data quality. These findings illustrate the immense challenge of implementing even modest replacements of paperbased systems that remain prevalent in hospital settings (Saleem et al., 2011; Waterson, 2014).

In addition to technical difficulties in implementing common IT projects, this study finds that 'socio-technical' issues appear to be more pronounced: that is, how IT 'fits' with working practices and how behaviour and wider organisational culture adapts (Greenhalgh et al., 2008). The loss of interpersonal contact proved of concern to some practitioners who valued speaking to the IDT member collecting the proformas, so as to emphasise or clarify certain points. There was also some evidence that electronic forms were then printed, returning to a paper-based system because meeting rooms did not have facilities to connect to the systems. These concerns are commonplace in the introduction of new electronic systems, and go some way to explaining how paper systems remain persistent across care networks (Saleem et al., 2011). The recommended solution is to view the introduction of new technologies as iterative cycles, requiring continual evaluation and improvements (Waterson, 2014).

\section{Limitations}

The curtailment of the implementation period to just five weeks means that the study was blind to how the module may have 'bedded-in' had it remained operational. It is likely that practitioners would have adapted to its use and identified possible 
'workarounds' (Saleem et al., 2011) to avoid some of its negative features - yet this remains speculation. That only 22 patients were referred to the IDT using the module is particularly problematic, since it limited the extent to which care planning and discharge outcomes could be monitored. Moreover, the research lacks a service user 'voice': patient satisfaction with the assessment processes and their care plans could have been evaluated, had the opportunity arisen. Finally, this paper presents a single case study from a larger suite of CAF initiatives. It was, however, unique in that it involved primary data collection; others employed secondary data analysis $(\mathrm{DH}$, 2012).

\section{Conclusions}

The case study found that an electronic discharge system may, in principle, have improved the efficiency and suitability of integrated care planning. Benefits may include a faster, more accurate and more secure means of data sharing. A key learning from the research was that project teams must pay attention to sociooccupational issues that lie beyond the physical and technical infrastructure of the project. The many challenges that such programmes encounter can easily distract project teams from exploring in full how end-users interact with the new technology. Yet the success of any shared assessment innovation will hinge on how well busy practitioners are able to integrate new processes within their working environment. The study also highlighted problems of scale, whereby relatively local IT projects can become overwhelmed by wider organisational decisions. The early closure of the initiative meant that longer-term outcomes remain unknown, and further research is needed to demonstrate whether IT projects to facilitate common assessments have sustainable benefits. 


\section{References}

Abell, J., Hughes, J., Reilly, S., Berzins, K. and Challis, D. (2010) Case management for long-term conditions: developing targeting processes, Care Management Journals, 11, 1, 11-8.

Abendstern, M., Clarkson, P., Challis, D., Hughes, J., and Sutcliffe, C. (2008) Implementing the Single Assessment Process for older people in England: lessons from the literature. Research, Policy and Planning, 26, 1, 15-31.

Byles, J. (2007) A thorough going over: evidence for health assessments for older people, Australian and New Zealand Journal of Public Health, 24, 2, 117-23.

Challis, D. and Davies, B. (1986) Case Management in Community Care: An Evaluated Experiment in the Home Care of the Elderly, Gower, Aldershot.

Challis, D., Abendstern, M., Clarkson, P., Hughes, J., and Sutcliffe, C. (2010) Comprehensive assessment of older people with complex care needs: the multidisciplinarity of the Single Assessment Process in England. Ageing and Society, 30, 7, 1115-1134.

Clarkson, P., Abendstern, M., Sutcliffe, C., Hughes, J., and Challis, D. (2009). Reliability of needs assessments in the community care of older people: impact of the single assessment process in England. Journal of Public Health, 31, 4, 521-529.

Chester, H., Hughes, J., Clarkson, P., Davies, S., \& Challis, D. (2015). Approaches to information sharing and assessment: evidence from a demonstration programme. Care Management Journals, 16, 3, 150-158.

Department of Health (DH) (2001) National Service Framework for Older People: Modern Standards and Service Models, Department of Health, London. 
Department of Health (DH) (2009) Common Assessment Framework for adults: A Consultation on Proposals to Improve Information Sharing around Multi-disciplinary Assessment and Care Planning, Department of Health, London.

Department of Health (DH) (2012) The Power of Information: Putting All of Us In Control of the Health and Care Information We Need, Department of Health, London.

Department of Health (DH) (2013) Making Sure Health and Social Services Work Together, Department of Health, London,

Department of Health \& Department for Communities and Local Government (DH \& DCLG) (2014) Better Care Fund: Policy Framework. Department of Health, London.

Dickinson, A. (2006) Implementing the Single Assessment Process: Opportunities and challenges, Journal of Interprofessional Care, 20, 4, 365-379.

Greenhalgh, T., Stramer, K., Bratan, T., Byrne, E., Mohammad, Y. and Russell, J. (2008) Introduction of shared electronic records: multi-site case study using diffusion of innovation theory. British Medical Journal, 337, 1-10.

Raiff, N. and Shore, K. (1993) Advanced Case Management: New Strategies for the Nineties, Sage Publications, London.

Saleem, J., Russ, A., Neddo, A., Blades, P., Doebbeling B., and Forsmann, B. (2011) Paper persistence, workarounds, and communication breakdowns in computerized consultation management. International Journal of Medical Informatics, 80, 466-479.

Scott, J., and Hawkins, P. (2008) Organisational silos: affecting the discharge of elderly patients. Journal of Health Organization and Management, 22, 3, 309-318.

Scottish Government (2013) Scotland's national dementia strategy: 2013-2016, Scottish Government, Edinburgh. Available at: http://www.gov.scot/Topics/Health/ 
Services /Mental-Health/Dementia/DementiaStrategy1316 [Accessed 20th November 2016]

Taylor, B. (2012) Development an integrated assessment tool for the health and social care of older people. British Journal of Social Work, 47, 7, 1293-1314.

Think Local Act Personal (2014) Delivering Care and Support Planning: Supporting Implementation of the Care Act 2014 Think Local Act Personal, London.

Van der Meijden, M.J., Tange, H.J., Troost, J. and Hasman, A. (2003) Determinants of success of inpatient clinical information systems: a literature review, Journal of Medical Informatics Association, 10, 3, 235-243.

Waterson, P. (2014). Health information technology and sociotechnical systems: A progress report on recent developments within the UK National Health Service (NHS) Applied Ergonomics, 45,2A, 150-161. 